\title{
INTERSECCIONES ENTRE LIDERAZGO Y FEMINISMO
}

\section{INTERSECTIONS BETWEEN LEADERSHIP AND FEMINISM}

Sonia Reverter-Bañón'

Universitat Jaume I

Maria Medina-Vicent ${ }^{2}$

Universitat Jaume I

En el volumen Mujeres y liderazgo que aquí presentamos ${ }^{3}$, ofrecemos una reflexión sobre el liderazgo de las mujeres en un sentido amplio, ya que la relación de aportaciones que conforman este número de Dossiers Feministes es variada en el enfoque, y ello, lejos de dispersar el concepto de liderazgo nos ayuda a comprenderlo de una forma más completa. Este monográfico nos permite, así, ver cómo en el liderazgo están interrelacionados aspectos como el talento, el empoderamiento identitario, la creatividad, o la cultura de comunicación y de conocimiento entre otros. A su vez, el recorrido sobre nuestra temática se hace transitando áreas de pensamiento y realidades muy distintas: desde los ámbitos empresarial, económico, educativo y tecnológico hasta los ámbitos de reflexión sobre el cuerpo.

En este recorrido intentamos reflexionar sobre una de las consignas feministas más claras y urgentes desde los años setenta: acabar con la ruptura dicotómica de los espacios público y privado. Son los conceptos de liderazgo, talento y empoderamiento estudiados en este número los que abren dicha posibilidad. Por ejemplo, el empoderamiento de una mujer a la hora de ejercer su liderazgo en una empresa puede estar conectado con las representaciones y experiencias de lactancia cuando acaba de ser madre. Como vemos, lo

\footnotetext{
1 Sonia Reverter-Bañón y Maria Medina-Vicent se encuentran dentro del Proyecto de Investigación Científca y Desarrollo Tecnológico FFI2016-76753-C2-2-P, financiado por el Ministerio de Economía y Competitividad del Gobierno de España. Contacto: reverter@ uji.es / medinam@uji.es

2 Además, Maria Medina-Vicent también trabaja dentro del Proyecto de Investigación Científca y Desarrollo Tecnológico UJIA2016-04, fnanciado por el Plan de Promoción de la Investigación de la Universitat Jaume I.

3 Este trabajo se enmarca dentro del Proyecto de Innovación Educativa 326/16 de la Universitat Jaume I.
} 
privado y lo público se entremezclan; no se confunden, pero están claramente imbricados. Y ello es más claro si cabe en el caso de las mujeres, como se encargó de argumentar Carole Pateman (1988) cuando nos desveló el contrato sexual como subtexto del contrato social.

El objetivo principal de este monográfico es, por ello, ayudar a ofrecer una visión que nos acerque a comprender la complejidad de entender el liderazgo desde la dimensión del género, a la vez que aportar elementos críticos que nos permitan desmontar los mitos simplistas que de forma inapropiada surgen de una valoración del liderazgo como una capacidad individual a «entrenar» derivada de los discursos actuales del management (Fernández-Rodríguez y Medina-Vicent, 2017).

En el análisis del concepto de liderazgo nos encontramos con reflexiones clásicas, como las de Platón, Sun Tzu, o Maquiavelo, o ya en el siglo XX, Max Weber, quienes nos han hablado de las funciones del líder (así, en masculino), e incluso de los diferentes tipos de líder (también claramente masculinizados). Este modelo de liderazgo sin adjetivos, nos habla, sin embargo, de un líder adjetivado: un líder masculino (y podríamos añadir blanco, occidental y enriquecido). Este discurso pretendidamente universal de liderazgo no será cuestionado hasta hace unas décadas. Si bien los tipos de liderazgo ya habían sido relacionados a veces con la feminidad y la masculinidad respectivamente, lo habían sido desde la perspectiva de valores femeninos o valores masculinos según una percepción totalmente acrítica de reunir los aspectos de debilidad, delicadeza, escucha, emotividad, como femeninos (Rosener, 1990; Helgesen, 1995; Helgesen y Johnson, 2010); y los aspectos de fortaleza, agresividad, asertividad, racionalidad, como masculinos; aproximación que reproduce los viejos estereotipos de género (Medina-Vicent, 2015a).

En todo caso el líder, que habrá de emplear estratégicamente esas características catalogadas como femeninas o masculinas, es siempre un varón. La feminidad o masculinidad se han ofrecido usualmente como características identitarias de mujeres u hombres (vinculadas, por tanto, al sexo), que pueden en cierta dosis ser imitadas por los líderes en beneficio de mejorar estratégicamente sus liderazgos. Hasta hace bien poco las mujeres no han sido propuestas como líderes, aunque algunas de sus características se han podido ofrecer como ayuda para gestionar y llevar adelante el rol de líder, sobre todo en contextos de crisis, cuando el think manager-think male se transforma en think crisis-think female (Ryan y Haslam, 2007). Es decir, cuando las cosas se ponen feas, lo más probable es que las capacidades asociadas al estereotipo de género femenino sean las que más rápidamente se considere que puedan cambiar el rumbo de la empresa hacia el éxito, y sacarla de la situación de crisis. Por esta razón, muchas empresas en crisis optan por situar a una mujer en cargos de responsabilidad, con la esperanza de que cambie el estilo de liderazgo previo y así puedan 
recuperarse. Cabe señalar que dichas cualidades suelen asociarse a un tipo de liderazgo de corte más transformacional, a diferencia del que se asocia al estereotipo masculino, que sería transaccional. Tal y como señalan los trabajos de Barberá, «la investigación psicológica ha tendido a relacionar el estilo transaccional con los estereotipos y roles masculinos, mientras que las características del estilo transformacional presentan mayor correspondencia con los roles estereotipados femeninos» (Barberá et al., 2011 : 176).

Es típico, por tanto, hablar de forma acrítica en algunos estudios de gestión del liderazgo de la conveniencia de "feminizar» aspectos para potenciar un liderazgo más provechoso, o más efectivo (Medina-Vicent, 2015b). Sin duda el objetivo de la inclusión de las características "femeninas» que el patriarcado dictamina es, normalmente, servir a los intereses de la productividad de un sistema neoliberal que mercantiliza incluso aspectos identitarios (Scharff, 2016). Así, lo femenino o lo masculino circulan en muchos discursos de liderazgo como mercancía que pueda usarse para incrementar la productividad. Al hablar hoy de nuevos liderazgos no deberíamos caer en esa trampa.

El concepto de género que la segunda ola del feminismo ofreció para entender y desenmascarar la desigualdad entre mujeres y hombres proporcionó a todas las áreas del conocimiento una posibilidad de integrar una reflexión crítica sobre cómo producen conocimiento y cómo organizan el mismo. Así, la introducción de la perspectiva de género en la economía y el trabajo permitió valorar hasta qué punto el conocimiento que producen y que vertebra nuestras vidas está diseñado para producir y mantener la desigualdad entre individuos, y muy especialmente entre mujeres y hombres. La inclusión de la perspectiva de género en estos ámbitos en concreto ha permitido transformar no sólo cómo entendemos la economía y el trabajo, sino cómo afectan a nuestras vidas. Y es que, la economía y el trabajo no son los mismos si se conciben desde el modelo desarraigado del «homo economicus» o desde el modelo de «homo socialis», que promueve dar valor de igualdad a las personas y sus interrelaciones frente a la mercancía.

De la misma manera, la introducción de la perspectiva de género en los espacios educativos puede servir para hacernos conscientes de la oportunidad que el espacio educativo, en todos sus niveles, ofrece como espacio privilegiado de cambio: de ensayarlo en sus estructuras propias y de promoverlo a través del proceso de enseñanza-aprendizaje con el estudiantado. Estas transformaciones han terminado por afectar cómo entendemos y proponemos el liderazgo, el talento o el empoderamiento.

Ahora bien, si desde la teoría feminista la introducción de la perspectiva de género a todos los campos posibles de conocimiento ha permitido visualizar desigualdades e injusticias, no es menos cierto que la solidificación del concepto de género ha permitido al 
patriarcado seguir instalando su tiranía hacia el colectivo de mujeres. Hablar de liderazgo y talento según características «femeninas» o «masculinas» es producto de esa solidificación del género que aquí criticamos. La razón última es que impide la promoción de valores que desde una renovada visión de lo que entendamos por liderazgo (o talento) pueda transformar las circunstancias de opresión, desigualdad e injusticia.

Por todo ello, desde la teoría feminista se vienen proponiendo modelos críticos de liderazgo que puedan superar estas argucias conceptuales del patriarcado en el uso del concepto de género (Due Billing y Alvesson, 2000; Lipton, 2015; Bierema, 2016). Aunque en las últimas tres décadas los llamados «Leadership Studies» han integrado de forma clara la perspectiva de género, sigue siendo usual la falta de una perspectiva crítica que promueva mayores implicaciones con la igualdad de género en las empresas y organizaciones. Como bien señalan algunas voces (Learmonth y Morrell, 2016) la transformación exige prácticas discursivas más críticas que las que gran parte de los estudios de liderazgo proponen. El riesgo de no hacerlo es acabar reproduciendo el modelo de liderazgo que condenamos, y que no es otro que el del «homo economicus» al que nos hemos referido. Teniendo en mente esta alerta abordamos la reflexión que nos congrega en este volumen.

Para comenzar, José María Biedma Ferrer nos alerta de la aún baja presencia de las mujeres en los consejos de administración de las compañías del lbex 35 . El problema no es el incumplimiento, una vez más, del porcentaje recomendado (no obligado) en la normativa española vigente, que es del $40 \%$, sino la pérdida de talento que ello pueda suponer. $\mathrm{Si}$ bien, y como ya hemos comentado, no se trata de buscar la «beneficiosa» diversidad que el género puede ofrecer a un mundo en el que la competitividad es creciente, sino de acometer las brechas que la escasa presencia de las mujeres en las estructuras de poder revela. Más que una cuestión de representación es una cuestión de justicia. Por ello, el autor nos alerta en su conclusión que habrá que estar atentas a la evolución de la presencia de las mujeres en puestos de responsabilidad en empresas del lbex 35 .

Así pues, son los sectores de más concentración de poder en los que de forma visible se percibe esa brecha en los puestos de toma de decisión y liderazgo. El sector de las tecnologías de la información y la comunicación es un claro ejemplo de dicha infrarrepresentación de las mujeres, como nos cuenta Laura Martínez Álvaro en su artículo. La autora, en busca de las causas que lo puedan explicar, no tiene duda en denominar esta situación como «digitalización del patriarcado», dando así por obvia la razón principal de la desigualdad de poder en el sector de las TIC. Ello muestra que los estereotipos de género tienen buena alianza con una cultura organizacional que es claramente opuesta a la estrategia de retención del talento femenino e igualdad de oportunidades. Como nos dice 
la autora «la cultura organizativa promueve una única medida, un único modelo, el de los hombres, como referencia de éxito en la organización».

De ahí la importancia para los feminismos de comprender los posibles procesos de empoderamiento y liderazgo. La necesidad de transformación social pasa por cambios culturales que desplacen los rasgos patriarcales (y coloniales) de las culturas. Silvia Martínez Cano nos propone estrategias de sororidad y creatividad para superar las alianzas de «fratriarquía», que establecen los varones. Este «gobierno de los hermanos», como nos cuenta la autora, controla social y culturalmente nuestras vidas: los comportamientos, las creencias y pensamientos, los cuerpos, las relaciones. Como resistencia Silvia Martínez Cano nos ofrece las estrategias que ya en la mitología griega encontramos: autoestima, asertividad, autonomía personal, insumisión... Todo ello nos ha de guiar hacia un liderazgo más solidario y creativo que sea capaz finalmente de llevarnos a pensar el concepto de política desde otros parámetros nuevos para la convivencia e interrelación social.

La responsabilidad de las universidades en este empoderamiento es crucial, ya que en sus objetivos está la formación para la libertad. Si bien la universidad se presenta, de nuevo, como un espacio de concentración de poder y de toma de decisiones de una sociedad determinada, también se nos presenta, como nos cuenta María Amparo Calabuig Puig en su artículo, «como esfera privilegiada desde la cual debemos proyectar los valores del empoderamiento en igualdad de condiciones al resto de la sociedad. Teniendo la misma una especial responsabilidad como agente socializador, trasmisor de valores y referente del pensamiento crítico». En este sentido, la autora nos relata la experiencia del «Taller Permanente de Oratoria y Debate», que realizaron en la Universidad Miguel Hernández de Elche el curso 2015/2016, como muestra de acciones de empoderamiento de las mujeres conducentes a un liderazgo que supere los roles tradicionales de género y permita la transformación social para un mundo más justo e igual. La universidad se revela como un lugar de poder, no sólo para reproducir patrones sexistas y de cultura patriarcal, sino también para subvertir estos patrones y crear modelos de progreso social, respeto e igualdad.

Desde las estructuras de poder y a través de jerarquías y liderazgos patriarcales se ejerce un control y sometimiento no solo sobre la consciencia, sino también en el cuerpo y con el cuerpo. Por ello, y como Foucault (2007) indicó, para la sociedad capitalista la biopolítica se convierte en una forma de gobierno prioritaria. El gobierno de los procesos biológicos, - biopolítica, es esencial para forjar la expansión del capitalismo liberal. Ese control es ejercido de manera desaforada sobre el cuerpo de las mujeres (Repo, 2016). En el presente volumen nos acercamos también a la biopolítica sobre el cuerpo de las mujeres en diferentes manifestaciones: gestación, parto, lactancia, aborto y trabajo sexual. 
Eva Margarita García nos cuenta en su artículo precisamente cómo la patologización y medicalización del cuerpo de las mujeres es una forma de «secuestro» por parte de la biomedicina. Ésta, con protocolos médicos que aseguran a los profesionales médicos como los únicos sujetos agentes en relación a los cuerpos de las mujeres, los cuáles son visto como cuerpos pasivos, desapropian a las mujeres de uno de los procesos más significativos y más personales en sus vidas: el parto. Este secuestro, origen de un verdadero relato de violencia obstétrica, apunta a un desempoderamiento en el mismo cuerpo de las mujeres. La vida y el cuerpo de las mujeres se convierten en objetos administrados y gestionados desde una ideología patriarcal dirigida por poderes, talentos y liderazgos formateados en la hegemonía androcéntrica, represora e impositiva.

No sólo la gestación y el parto están altamente controlados y medicalizados por la biomedicina, sino que, como nos relatan $M^{a}$ Dolores Pérez Bravo y Amparo Moreno Hernández, la lactancia también está secuestrada por un discurso bio-social que decide e impone a las mujeres, según el contexto histórico, social y cultural, si hay que amamantar o no a infantes y hasta cuándo y cómo hacerlo. Esta des-territorialización del cuerpo de las mujeres es sintomática y extrema en el caso de la violación que además produce un embarazo en las mujeres violadas.

En este caso, y como nos cuenta Meysis Carmenati González a propósito del aborto en Ecuador, la penalización del aborto supone la negación de cualquier derecho y reconocimiento a la mujer. Como menciona la autora, en este caso se da «la existencia de dispositivos políticos sobre los cuerpos de las mujeres, junto a la obligatoriedad de la maternidad como filtro por donde se reconoce, especialmente, la identidad de las mujeres y su deber en la reproducción social». La violencia como forma de control y anulación del sujeto se desarrolla así en un cuádruple escenario de desapropiación y alienación sobre el propio cuerpo: violación, embarazo, aborto y penalización del aborto. La degradación de la vida en estas circunstancias apunta a sistemas de gobierno de la vida (jurídicos, médicos, políticos, culturales...) que conllevan prácticas cotidianas de violencia contra las mujeres.

En este campo de ruina humana, y como las diferentes autoras de los textos presentados nos explican, resulta interesante entender los procesos que acarrean una labor de desmantelamiento de esta hegemonía: la sororidad, los lazos entre los sectores sin poder, el asociacionismo de grupos de mujeres que permitan visibilizar la opresión y desarticularla. Resulta interesante en este contexto el análisis que Elena Martínez Pérez hace en relación a las trabajadoras sexuales y cómo estas han podido articular un discurso de empoderamiento en el espacio público a través de diferentes estrategias y alianzas con otros colectivos sociales. Las soluciones individuales a la opresión, son, como Zillah Eisenstein (2007) nos dice, un camino que nos llevará antes a aliarnos con el capitalismo que a derribarlo. 
Esta tarea de creación de alianzas y redes supone, no sólo la posibilidad de contestación a la hegemonía patriarcal que colapsa los sentidos transformadores de liderazgo, sino una revisión de los propios presupuestos feministas, muchas veces co-optados por un capitalismo neo-liberal que ha convertido la «perspectiva de género» en una aliada en la gobernación de la vida. La alerta de feminismos «periféricos», como nos relata Daniel Peres Díaz en su artículo, nos puede servir como aviso para pensar un feminismo que no sea hegemónico y etnocéntrico. Lo que algunas autoras llaman la «neo-liberalización del feminismo» (Prügl, 2014) nos puede llevar a un feminismo que se ocupe de entrever los aspectos provechosos de un sistema económico que necesita integrar a las mujeres, también como líderes, para poder explotar su producción. En este camino el derribo por parte de mujeres individuales de los muros patriarcales ha sido muy visible en las últimas décadas; pero cuando la opresión y dominación de la mayoría sigue latente es porque las estructuras de dominio siguen, no se han relajado, sólo han abierto pequeños intersticios por los que, muchas veces a base de «negociar» los mandatos de género, se ha podido penetrar. La contestación de algunas mujeres visibiliza que el objetivo de llegar a ciertas metas es posible, pero también deja en evidencia que las estructuras que oprimen a la mayoría siguen estando ahí.

El escenario de transformación de las sociedades sólo es posible desde el nosotrxs. Si queremos que la transformación sea ética, es decir, asegurando la igualdad en dignidad de los seres humanos, es necesario trabajar y luchar desde la conexión que en la opresión y la precariedad podamos tener nosotros, vosotros, Ixs de aquí, Ixs de allá, Ixs próximxs, Ixs distantes. Como nos dice Judith Butler no tenemos elección: «Vivimos juntos porque no tenemos elección» (Butler, 2015, 122). Y en ese no tener elección se produce el esperanzador acontecimiento de poder luchar juntxs en un contexto globalizado que precisa repensar las dinámicas combativas del feminismo (Reverter-Bañón, 2017a, 2017b).

\section{Bibliografía}

BarberÁ, Esther, Ramos, Amparo y Candela, Carlos (2011): «Laberinto de cristal en el liderazgo de las mujeres», Psicothema. Vol. $23 \mathrm{~N}^{\circ} 2$, pp. 173-179.

BieremA, Laura (2016): «Women's Leadership. Troubling Notions off the «ldeal» (Male) Leader», Advances in Developing Human Resources. Vol. 18, № 2, pp. 119-136.

Butler, Judith (2015): Notes Towards a Performative Theory of Assembly, Boston, Harvard University Press. 
Due BILING, Yvonne y AlveSSON, Mats (2000): «Questioning the Notion of Feminine Leadership:

A Critical Perspective on the Gender Labelling of Leadership», Gender, Work \& Organization. Vol. $7 \mathrm{~N}^{\circ} 3$, pp. 144-157.

EISENSTEIN, Zillah (1993): The Radical Future of Liberal Feminism, Boston, Northeastern University Press.

Fernández-RodRíguez, Carlos y Medina-VICENt, Maria (2017): «Los nuevos discursos del management: impactos y tendencias», Recerca. Revista de Pensament i Anàlisi. № 20, pp. 7-14.

Foucault, Michel (2007): El nacimiento de la biopolítica, México, Fondo de Cultura Económica. Helgesen, Sally (1995): The Female Advantage: Women's Ways of Leadership, New York, Doubleday Currency.

Helgesen, Sally y Johnson, Julie (2010): The Female Vision: Women's Real Power at Work, New York, Berrett-Koehler Publishers.

Learmonth, Mark y MorRelL, Kevin (2016): «ls critical leadership studies 'critical'? », Leadership. Vol. $13 \mathrm{~N}^{\circ} 3$, pp. 257-271.

LIPTON, Briony (2015): «Writing through the labyrinth: Using l'ecriture feminine in leadership studies», Leadership. Vol. $13 \mathrm{~N}^{\circ} 1$, pp. 64-80.

Medina-VICENT, Maria (2015a): «Women's Leadership: An Essentialist Concept?», Journal of Feminist, Gender and Women Studies. № 2, pp. 69-72.

MEDINA-VICENT, Maria (2015b): «Aproximación al estudio del Liderazgo Femenino a través del modelo transformacional», en UNITAT D'IGUALTAT UJI: Investigació i gènere a la Universitat Jaume I, Castelló de la Plana, Publicacions de la Universitat Jaume I.

Pateman, Carole (1988): The Sexual Contract, Los Angeles, Stanford University Press.

PRÜGL, Elisabeth (2014): «Neoliberalising Feminism», New Political Economy. № 37, pp. 301-325. RePo, Jemina (2016): The Biopolitics of Gender, Oxford, Oxford University Press.

ReVerter-Bañón, Sonia (2017a): «Cosmopolitismo Feminista contra Globalización», Araucaria. Revista Iberoamericana de Filosofía, Política y Humanidades. №32, pp. 549-572.

Reverter-BAÑón, Sonia (2017b): «Performatividad: la teoría especial y la general», Isegoría.

Revista de Filosofía Moral y Política. N56, pp. 61-87.

Rosener, Judy (1990): Ways Women Lead, Boston, Harvard Business School.

RYAN, M. K. y HASLAM, S. A. (2007): «The glass cliff: Exploring the dynamics surrounding women's appointment to precarious leadership positions», Academy of Management Review. N³2, pp. 549-572.

ScharfF, Christina (2016): Repudiating Feminism: Young Women in a Neoliberal World, London and New York, Routledge. 